\title{
Alternative forms for the promoting interaction in radiationless transitions
}

\author{
R H Bartram $†$ and A M Stoneham \\ Theoretical Physics Division, AERE Harwell, Oxon OX11 ORA, UK
}

Received 18 April 1985

\begin{abstract}
Alternative forms for the radiationless transition rate in the adiabatic coupling scheme are derived, with a minimum of special assumptions, for the case of coupling to several distinct promoting and accepting coordinates. Their relation to the static coupling scheme is discussed. The resulting expressions are suitable for use with state-of-the-art electronic structure calculations, i.e. not merely model systems. The appropriate application of pseudopotential theory in this context is considered.
\end{abstract}

In the conventional description, radiationless transitions between adiabatic states are mediated by the kinetic energy operator (Huang and Rhys 1950, Lax 1952). However, several authors have advocated an alternative coupling scheme in which radiationless transitions between static states are mediated by off-diagonal elements of the potential energy operator (Helmis 1956, Pässler 1974, 1982). Since no radiationless transitions are possible between true stationary states, the choice ultimately depends on which nonstationary state is prepared in a given experiment (Bixon and Jortner 1969). It has been demonstrated recently by several authors (Huang 1981, Gutsche 1982, Burt 1983) that the two coupling schemes lead to equivalent predictions at a certain level of approximation, although they cannot be precisely equivalent (Denner and Wagner 1984). It has been demonstrated further that, within the adiabatic coupling scheme, the popular 'Condon' approximation introduced by Huang and Rhys (1950) is internally inconsistent, as usually applied, and predicts transition rates which are too small by three orders of magnitude.

Comparisons of the two coupling schemes have been presented within the context of a highly idealised model, involving a single configuration coordinate $Q$ and two static electronic states, which are assumed to provide a complete set for expansion of adiabatic states with $Q$-dependent coefficients. This idealised model is well adapted to clarification of matters of principle, but tends to obscure the procedures appropriate to more realistic models. Generalisation of the idealised model to encompass a larger basis set and several configuration coordinates was undertaken by Gutsche (1982).

The object of the present work is to extend these considerations to more realistic models. We proceed from a somewhat different set of assumptions than Gutsche (1982). In particular, we exploit a common feature of symmetrical systems: the distinction between promoting coordinates, which mix the symmetries of initial and final states,

+ Permanent address: Department of Physics and Institute of Materials Science, University of Connecticut, Storrs, CT 06268 , USA. 
and accepting coordinates, which absorb the excess electronic energy (Lin and Bersohn (1968). As a consequence, we do not require any restriction on the anharmonicity of accepting modes. Several alternative forms for the promoting interaction are presented, all derived within the adiabatic coupling scheme with a minimum of special assumptions, by a method which does not rely on expanding adiabatic wavefunctions in a static basis. These forms should prove convenient in calculations with detailed many-electron wavefunctions of the sort obtained from state-of-the-art electronic structure codes, coupled to several configuration coordinates. Incidentally, these alternative forms provide still another demonstration of the approximate equivalence of adiabatic and static coupling schemes.

In the adiabatic coupling scheme, radiationless transitions are presumed to occur between Born-Oppenheimer states $\Psi_{\text {n } \nu}(\nu, \theta)$, given by

$$
\Psi_{\mathrm{n} \nu}(r, Q)=\varphi_{\mathrm{n}}(r, Q) \theta_{\mathrm{n} \nu}(Q)
$$

where $\varphi_{\mathrm{n}}(r, Q)$ is an eigenfunction of the electronic Hamiltonian $H_{\mathrm{e}}(Q)$ for fixed nuclear coordinates $Q$,

$$
\begin{aligned}
& H_{\mathrm{e}}(Q)=T_{\mathrm{E}}+V(r, Q) \\
& H_{\mathrm{e}}(Q) \varphi_{\mathrm{n}}(r, Q)=U_{\mathrm{n}}(Q) \varphi_{\mathrm{n}}(r, Q) .
\end{aligned}
$$

The electronic eigenvalue $U_{\mathrm{n}}(Q)$ then serves as the potential energy of interaction of the nuclei

$$
\left[T_{\mathrm{N}}+U_{\mathrm{n}}(Q)\right] \theta_{\mathrm{n} \nu}(Q)=E_{\mathrm{n} \nu} \theta_{\mathrm{n} \nu}(Q) .
$$

In these equations, $r$ and $Q$ denote respectively all electronic coordinates and all symmetry-adapted combinations of nuclear coordinates, and $T_{\mathrm{E}}$ and $T_{\mathrm{N}}$ are the respective electronic and nuclear kinetic energy operators.

Radiationless transitions between Born-Oppenheimer states are mediated by the non-adiabaticity operator $H_{\mathrm{NA}}$, defined by

$$
H_{\mathrm{NA}} \Psi_{\mathrm{n} \nu}(r, Q)=\left[T_{\mathrm{N}}, \varphi_{\mathrm{n}}(r, Q)\right] \theta_{\mathrm{n} \nu}(Q) .
$$

The operator $H_{\mathrm{NA}}$ can be expressed as a sum over symmetry-adapted nuclear coordinates $Q$, and the promoting coordinates $Q_{\mathrm{p}}$ are just those for which matrix elements of $H_{\mathrm{NA}}$ are non-vanishing for the electronic states of interest. It is convenient to assume that $\varphi_{\mathrm{n}}(r, Q)$ depends only linearly on $Q_{\mathrm{p}}$, which is equivalent to neglecting $\partial^{2} \varphi_{\mathrm{n}} / \partial Q_{\mathrm{p}}^{2}$; in many cases this follows from symmetry alone. No such assumption is made concerning accepting coordinates $Q_{\mathrm{a}}$ since, for them, large displacements from equilibrium associated with avoided crossings of adiabatic potential energy surfaces are of special importance in the theory.

The radiationless transition rate, in first-order time-dependent perturbation theory, is given by

$$
\begin{aligned}
& W_{\mathrm{NR}}=(2 \pi / \hbar) \sum_{\alpha} \sum_{\beta} \sum_{\mathrm{p}} P_{\alpha}\left|\left\langle\theta_{\mathrm{f} \beta}\left|R_{\mathrm{p}}(\mathrm{f}, \mathrm{i} ; Q)\left[T_{\mathrm{N}}, Q_{\mathrm{p}}\right]\right| \theta_{\mathrm{i} \alpha}\right\rangle\right|^{2} \delta\left(E_{\mathrm{p} \beta}-E_{\mathrm{i} \alpha}\right) \\
& P_{\alpha}=\exp \left(-E_{\mathrm{i} \alpha} / k_{\mathrm{B}} T\right) / \sum_{\gamma} \exp \left(-E_{\mathrm{i} \gamma} / k_{\mathrm{B}} T\right) \\
& R_{\mathrm{p}}(\mathrm{f}, \mathrm{i} ; Q)=\left\langle\varphi_{\mathrm{f}}(Q)\left|\partial / \partial Q_{\mathrm{p}}\right| \varphi_{\mathrm{i}}(Q)\right\rangle .
\end{aligned}
$$

Equations (6)-(8) provide the point of departure for the derivation of several alternative forms below. 
We proceed by employing the identity

$$
\left[T_{\mathrm{N}}, Q_{\mathrm{p}}\right]=-\hbar^{2} \partial / \partial Q_{\mathrm{p}}
$$

in equation (6) to obtain

$$
W_{\mathrm{NR}}=(2 \pi / \hbar) \sum_{\alpha} \sum_{\beta} \sum_{\mathrm{p}} P_{\alpha}\left|\left\langle\theta_{\mathrm{f} \beta}\left|-\hbar^{2} R_{\mathrm{p}}(\mathrm{f}, \mathrm{i} ; Q) \partial / \partial Q_{\mathrm{p}}\right| \theta_{\mathrm{i} \alpha}\right\rangle\right|^{2} \delta\left(E_{\mathrm{p} \beta}-E_{\mathrm{i} \alpha}\right) \text {. }
$$

Equations (10) and (8) together comprise the first form. The quantity $R_{\mathrm{p}}(\mathrm{f}, \mathrm{i} ; Q)$ can be evaluated for a range of values of coordinates $Q$, by performing the required overlap integrals in the approximate expression

$$
R_{\mathrm{p}}(\mathrm{f}, \mathrm{i} ; Q) \approx\left\langle\varphi_{\mathrm{f}}(Q) \mid \varphi_{\mathrm{i}}\left(Q+\Delta Q_{\mathrm{p}}\right)\right\rangle / \Delta Q_{\mathrm{p}}
$$

for a small finite value of $\Delta Q_{p}$; see Galloy and Lorquet (1977) and Hirsch et al (1980) for a comprehensive discussion of such integrals. The quantity $R_{\mathrm{p}}(\mathrm{f}, \mathrm{i} ; Q)$ is a sensitive function of the accepting coordinator, and is particularly enhanced near the avoided crossing of adiabatic potential energy surfaces. It remains to evaluate the integrals over configuration coordinates in equation (10) by some suitably chosen approximate procedure, e.g. Monte Carlo integration.

Another form is derived by differentiating both sides of equation (3) with respect to $Q_{\mathrm{p}}$ for the case $N=\mathrm{i}$, multiplying left by $\varphi_{\mathrm{f}}(r, Q)$, and integrating over electronic coordinates to obtain

$$
R_{\mathrm{p}}(\mathrm{f}, \mathrm{i} ; Q)=\left[U_{\mathrm{i}}(Q)-U_{\mathrm{f}}(Q)\right]^{-1} S_{\mathrm{p}}(\mathrm{f}, \mathrm{i} ; Q)
$$

where

$$
S_{\mathrm{p}}(\mathrm{f}, \mathrm{i} ; Q)=\left\langle\varphi_{\mathrm{p}}(Q)\left|\partial H_{\mathrm{e}} / \partial Q_{\mathrm{p}}\right| \varphi_{\mathrm{i}}(Q)\right\rangle .
$$

Equations (10), (12) and (13) together comprise the second form. Since only nuclear potential energy terms depend on $Q_{\mathrm{p}}$, only one-electron integrals are involved in the approximate expression

$$
S_{\mathrm{p}}(\mathrm{f}, \mathrm{i} ; Q) \approx\left\langle\varphi_{\mathrm{f}}(Q)\left|H_{\mathrm{e}}\left(Q+\Delta Q_{\mathrm{p}}\right)\right| \varphi_{\mathrm{i}}(Q)\right\rangle / \Delta Q_{\mathrm{p}} .
$$

This integral is expected to be relatively insensitive to $Q$, but the adiabatic potential energy functions are required in equation (12). Note that, in this form, the same potential energy term governs both the avoided crossing and the radiationless transition rate; no double counting is involved, however, since in the latter instance the term in question arises from a transformation of the nuclear kinetic energy operator. Again, one is left with an integral over configuration coordinates in equation (10).

An alternative transformation of the integral in equation (6) can be effected by employing closure, as follows:

$$
\begin{aligned}
\left\langle\theta_{\mathrm{f} \beta}\left|R_{\mathrm{p}}(\mathrm{f}, \mathrm{i} ; Q)\left[T_{\mathrm{N}}, Q_{\mathrm{p}}\right]\right| \theta_{\mathrm{i} \alpha}\right\rangle & \\
& =\sum_{\gamma}\left\langle\theta_{\mathrm{f} \beta}\left|R_{\mathrm{p}}(\mathrm{f}, \mathrm{i} ; Q)\right| \theta_{\mathrm{fy}}\right\rangle\left\langle\theta_{\mathrm{f} \gamma}\left|\left[T_{\mathrm{N}}, Q_{\mathrm{p}}\right]\right| \theta_{\mathrm{i} \alpha}\right\rangle .
\end{aligned}
$$

From equation (4) we obtain

$$
\begin{aligned}
& \left\langle\theta_{\mathrm{f} \gamma}\left|\left[T_{\mathrm{N}}, Q_{\mathrm{p}}\right]\right| \theta_{\mathrm{i} \alpha}\right\rangle \\
& \quad=\left\langle\theta_{\mathrm{f} \gamma}\left|\left[E_{\mathrm{f} \gamma}-E_{\mathrm{i} \alpha}-U_{\mathrm{f}}(Q)+U_{\mathrm{i}}(Q)\right] Q_{\mathrm{p}}\right| \theta_{\mathrm{i} \alpha}\right\rangle .
\end{aligned}
$$


Energy conservation requires that $E_{\mathrm{f} \beta}=E_{\mathrm{i} \alpha}$. Since the final state vibrational wavefunction $\theta_{\mathrm{f} \beta}$ is highly excited, and therefore much more rapidly varying than $R_{\mathrm{p}}(\mathrm{f}, \mathrm{i} ; Q)$, only a small range of intermediate states will be important and one can safely neglect $E_{\mathrm{f} \gamma}-E_{\mathrm{i} \alpha}$ compared with $U_{\mathrm{i}}(Q)-U_{\mathrm{f}}(Q)$ in equation (15). With this approximation, we obtain

$$
\begin{aligned}
& \left\langle\theta_{\mathrm{f} \beta} \mid R_{\mathrm{p}}(\mathrm{f}, \mathrm{i} ; Q)\left[T_{\mathrm{N}}, Q_{\mathrm{p}}\right] \theta_{\mathrm{i} \alpha}\right\rangle \\
& =\left\langle\theta_{\mathrm{f} \beta}\left|R_{\mathrm{p}}(\mathrm{f}, \mathrm{i} ; Q)\left[U_{\mathrm{i}}(Q)-U_{\mathrm{f}}(Q)\right] Q_{\mathrm{p}}\right| \theta_{\mathrm{i} \alpha}\right\rangle .
\end{aligned}
$$

Combination of equations (10), (12) and (17) yields

$$
W_{\mathrm{NR}}=(2 \pi / \hbar) \sum_{\alpha} \sum_{\beta} \sum_{\mathrm{p}} P_{\alpha}\left|\left\langle\theta_{\mathrm{f} \beta}\left|S_{\mathrm{p}}(\mathrm{f}, \mathrm{i} ; Q) Q_{\mathrm{p}}\right| \theta_{\mathrm{i} \alpha}\right\rangle\right|^{2} \delta\left(E_{\mathrm{f} \beta}-E_{\mathrm{i} \alpha}\right) \text {. }
$$

Equations (18) and (13) together comprise the third form. A similar transformation was employed by Peuker et al (1982), in the context of a single-coordinate model, where it was necessarily restricted to the Condon approximation.

In all three forms, one is left with a difficult integral over configuration coordinates. However, the relative insensitivity of $S_{\mathrm{p}}(\mathrm{f}, \mathrm{i} ; Q)$ in equation (18) encourages one to replace the configuration coordinates $Q$ in this expression by some arbitrarily selected set of constant values $Q_{0}$, such as their equilibrium values in the initial state, so that the factor $S_{\mathrm{p}}\left(\mathrm{f}, \mathrm{i} ; Q_{0}\right)$ can be removed safely from the integral. (Note that the corresponding substitution in $\left.R_{\mathrm{p}} F, \mathrm{i} ; Q\right)$ in equations $(10)$ and $(12)$, including the factor $\left[U_{\mathrm{i}}(Q)-\right.$ $\left.U_{\mathrm{f}}(Q)\right]^{-1}$, is the now discredited 'Condon' approximation (Huang 1981). If one makes the additional assumption that it is feasible to separate variables in equation (4), and that the chosen configuration coordinates are identical with the normal coordinates, then the vibrational wavefunctions may be written as

$$
\theta_{\mathrm{n} \nu}(Q)=\prod_{\mathrm{p}} \chi_{\mathrm{n} \nu_{\mathrm{p}}}\left(Q_{\mathrm{p}}\right) \prod_{\mathrm{a}} \chi_{\mathrm{n} \nu \mathrm{a}}\left(Q_{\mathrm{a}}\right)
$$

and equation (18) becomes

$$
\begin{aligned}
W_{\mathrm{NR}}=(2 \pi / \hbar) & \sum_{\mathrm{p}}\left|\left\langle\varphi_{\mathrm{f}}\left(Q_{0}\right)\left|\partial H_{\mathrm{e}} / \partial Q_{\mathrm{p}}\left(Q_{0}\right)\right| \varphi_{\mathrm{i}}\left(Q_{0}\right)\right\rangle\right|^{2} \\
& \times \sum_{\alpha} \sum_{\beta} P_{\alpha}\left|\left\langle\chi_{\mathrm{f} \beta_{\mathrm{p}}}\left|Q_{\mathrm{p}}\right| \chi_{\mathrm{i} \alpha_{\mathrm{p}}}\right\rangle\right|^{2} \prod_{\mathrm{a}}\left|\left\langle\chi_{\mathrm{f} \beta_{\mathrm{a}}} \mid \chi_{\mathrm{i} \alpha_{\mathrm{a}}}\right\rangle\right|^{2} \delta\left(E_{\mathrm{f} \beta}-E_{\mathrm{i} \alpha}\right) .
\end{aligned}
$$

Finally, if one adopts the harmonic approximation in order to facilitate the evaluation of integrals over vibrational wavefunctions the radiationless transition rate predicted by equation (20) becomes identical with that appropriate to the static coupling scheme. Equation (20) with the harmonic approximation, which is a simplification of our third form, is by far the easiest to use and will be preferred in most applications.

A word of caution is in order concerning the application of these alternative forms to model systems. Very often, electronic structure calculations rely on some sort of pseudopotential or frozen-core approximation, in which the requirement that valence orbitals be orthogonalised to occupied core orbitals is reformulated in terms of an effective potential. The formal development presented here would go through identically if $V(r, Q)$ were replaced by a pseudopotential and $\varphi_{\mathrm{n}}(r, Q)$ by a pseudowavefunction; however, the predicted radiationless transition rates would be very different. The discrepancy arises because different physical assumptions are involved in the two cases.

Radiationless transitions between adiabatic states are manifestations of the failure of the electronic wavefunction to adjust to the instantaneous positions of the nuclei. In 
the pseudopotential approximation, the frozen cores are assumed to follow the nuclei with no difficulty, and only the valence pseudo-wavefunctions do not. Accordingly, the radiationless transition rate is grossly underestimated. In our first form, this underestimate arises from the smoothness of the pseudo-wavefunction, which diminishes the value of the integral in equation (8). In our third form, it arises primarily from the weakness of the pseudopotential in comparison with the nuclear potential. In any case, it is clear that one must orthogonalise the pseudo-wavefunction to occupied core orbitals before employing any of the formulae presented here for the calculation of radiationless transition rates-the situation is analogous to the 'Adrian effect' in the calculation of spin-Hamiltonian parameters (Adrian 1957).

This work was supported in part by GTE Laboratories Inc, and in part by the US Army Research office under Contract DAAG29-82-K-0158. R H Bartram gratefully acknowledges the hospitality of the Theoretical Physics Division, AERE, Harwell.

\section{References}

Adrian F J 1957 Phys. Rev. 1061356

Bixon M and Jortner J 1969 J. Chem. Phys. 504061

Burt M G 1983 J. Phys. C: Solid State Phys. 164137

Denner V and Wagner M 1984 J. Phys. C: Solid State Phys. 17153

Galloy C and Lorguet J C 1977 J. Chem. Phys. 674672

Gutsche E 1982 Phys. Status Solidi b109 583

Helmis G 1956 Ann. Phys., Lpz 1941

Hirsch G, Bruna P J, Buenker R J and Peyerimhoff S D 1980 Chem. Phys. 45335

Huang K 1981 Sci. Sinica 2427

Huang K and Rhys A 1950 Proc. R. Soc. A204 406

Lax M 1952 J. Chem. Phys. 201752

Lin S H and Bersohn R 1968 J. Chem. Phys. 482732

Pässler R 1974 Czech. J. Phys, B24 322

- 1982 Czech. J. Phys. B32 246

Peuker K, Enderlain R, Schenk A and Gutsche E 1982 Phys. Status Solidi b109 599 